\title{
An overview of Algorithms for Adaptive Beam Forming (ABF) for Smart Antenna System (SAS)
}

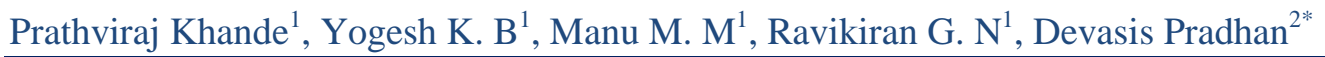

${ }^{\mathrm{T}}$ Final Year U.G Students, Department of Electronics \& Communication Engineering, Acharya Institute of Technology, Bengaluru - 560107, Karnataka, India

${ }^{2}$ Assistant Professor, Department of Electronics \& Communication Engineering, Acharya Institute of Technology, Bengaluru -560107, Karnataka, India

\section{*Corresponding Author}

Devasis Pradhan

\section{Article History}

Received: 13.01.2021

Accepted: 25.01.2021

Published: 30.01.2021

\begin{abstract}
The quantity of cellular customers are increasing rather all over the world. It's far necessary to growth the channel bandwidth and capacity and on the identical time minimize the channel interference. Smart antennas are considered as an effective counter measure to achieve these necessities because they provide extensive bandwidth, less electromagnetic interference, flexibility, less weight, excessive velocity, phase control unbiased of frequency and coffee propagation loss. Smart antennas integrate the antenna array with sign processing to optimize mechanically the beam pattern in reaction to the acquired signal. Beam forming may be used for both radio or sound waves; it has determined numerous programs in radar, sonar, seismology, wireless communications, radio astronomy, speech and bio-medicine. This paper discuss the algorithm for blind and non blind adaptive beam forming technique for reliable wireless communication.
\end{abstract}

Keywords: Beam forming, Adaptive beam forming, Blind algorithm and Non Blind algorithm, SAS, AF.

\section{INTRODUCTION}

As the growing call for for mobile communications is constantly increasing, the need for better coverage, advanced capability, and better transmission best rises. For this reason, a extra green use of the radio spectrum is required. Beam forming systems are able to successfully utilizing the radio spectrum and are a promise for an powerful approach to the existing wi-fi systems issues at the same time as reaching reliable and strong high-speed, excessiveinformation-fee transmission. The algorithm then makes use of this information from a user by comparing each received signal to the original sequence to find out the correct radiation pattern for that user. With this method, all received signals from each antenna element are used and are optimally combined to enhance the desired signal and to cancel unwanted interference.

Copyright (C) 2021 The Author(s): This is an open-access article distributed under the terms of the Creative Commons Attribution 4.0 International License (CC BY-NC 4.0) which permits unrestricted use, distribution, and reproduction in any medium for non-commercial use provided the original author and source are credited. 


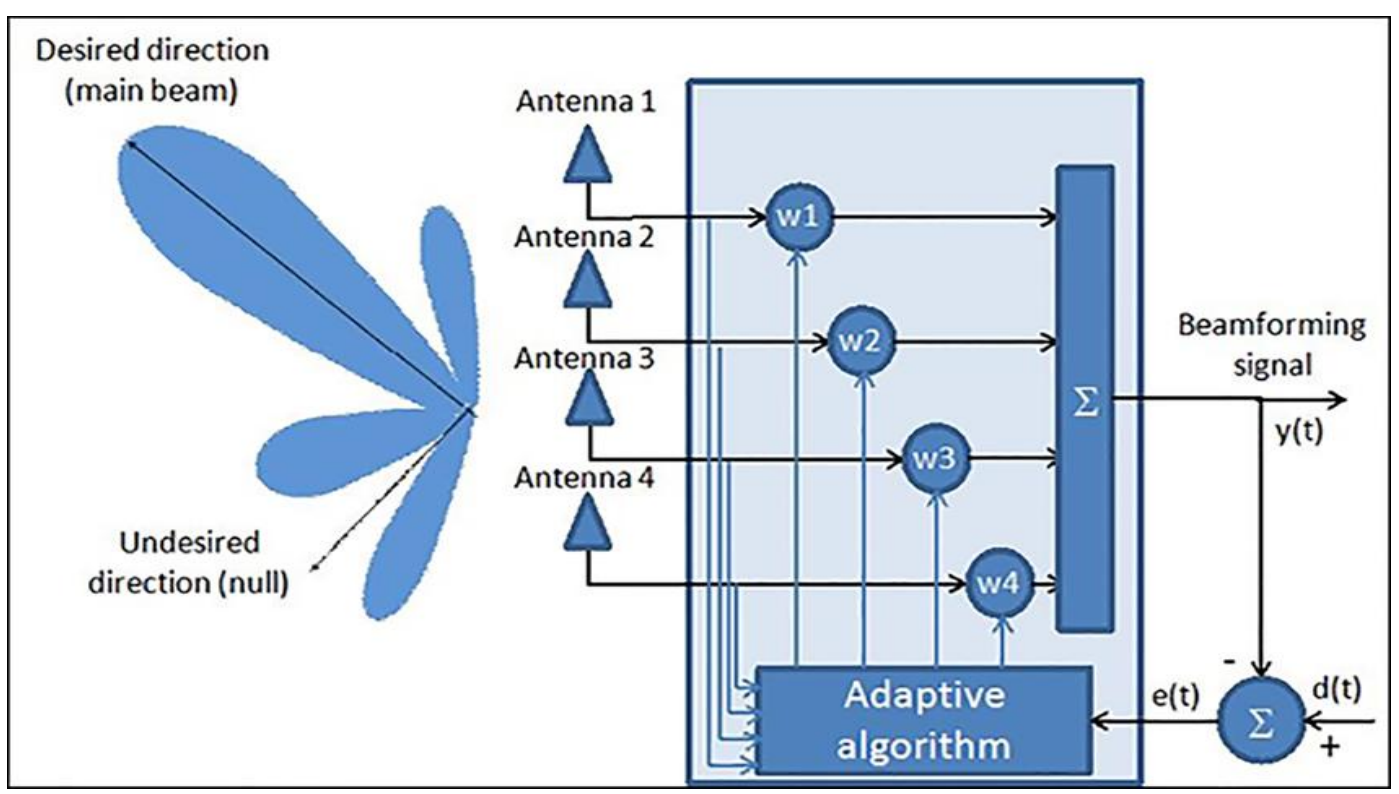

Fig-1: Functional block diagram for beam former at the receiver

The usage of an antenna array adds an extra size and makes the usage of spatial diversity feasible. This is because of the reality that the interference hardly ever have the equal geographical region because the person and therefore they are spatially separated. Special from omnidirectional antenna device and sectored antenna machine, adaptive antenna array (clever Antenna) device combine an antenna array and a virtual signal processor to acquire and transmit alerts in a directional manner.

Consequently the beam sample at the bottom station may be adaptively modified. The main function of antennas is to effectively transmit and receive the radio signals, there are some additional functions that smart antennas are required to accomplish which are:

A. Direction of arrival [DOA] estimation: In order to provide optimized transmission and reception, antennas are required to detect the direction of arrival of the required incoming signal. This information is then transferred to signal processor present within the antenna and required analysis is provided by the processor.

B. Beam forming: After the analysis from DOA estimation, the circuitry inside the antenna is able to optimize the beam pattern in certain required direction to provide the required performance. Beam forming is a technique which involves utilization of array of antennas to attain a wireless signal towards a specific direction, rather than having the signal spread in all directions as it normally would and the signals which have same frequency from different directions are abandoned.

Beam forming is the main function on which this paper mainly centralizes. Beam forming as the name illustrate provides narrow beam in desired location in addition to that it also reduces interference. If the angle of arrival is constant then fixed beam forming is used but if the angle of arrival changes continuously with respect to time then adaptive beam forming comes into the picture.

\section{Smart Antenna System (SAS)}

The smart antennas structures obtain better potential boom in evaluation with the switched beam structures especially in the case of densely populated insurance areas and reduce more efficaciously the terrible impacts of the interference. Moreover, there are more benefits that may be counted in prefer of adaptive array structures along with variety growing, protection enhancement (greater tough to tap any connection) [1], and place-based offerings improvements especially for emergency situations (spatial detection characteristics). Figure-2 shows the smart antenna system (SAS). 


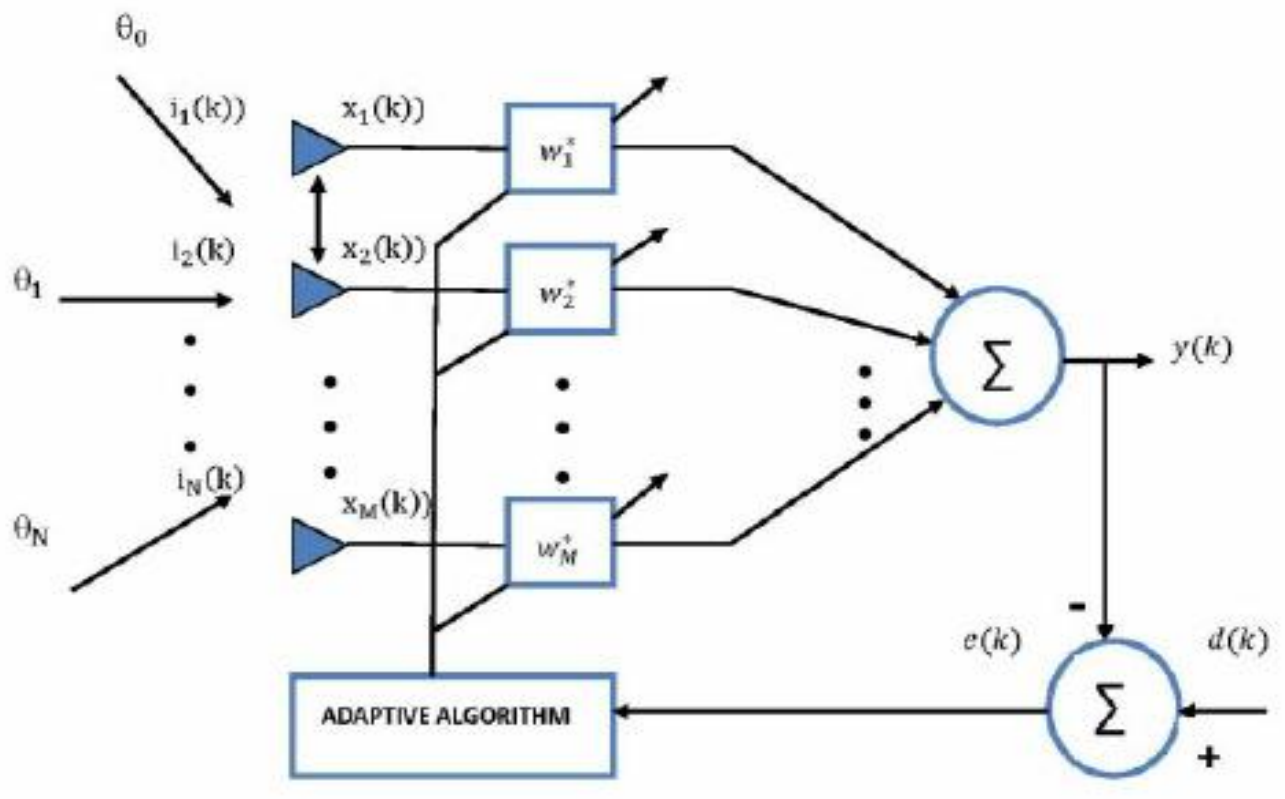

Fig-2: Block diagram for SAS

As inside the case of any system or generation, some disadvantages or drawbacks of the smart antenna structures are discovered just like the complexity of transmitters and receivers design, the excessive computation intensity with the need of effective digital signal processors (DSPs), and the ordinary machine employment value.

Hence, the smart antennas systems relay on the adaptive signal processing strategies inclusive of DOA estimation and adaptive beam forming under using more than one antenna configurations (antenna arrays). Right here, it's far very beneficial to make a few feedback about the importance of antenna arrays inside the improvement of the preceding concepts.

\section{Benefits of Smart Antenna System (SAS)}

Smart antenna system has executed a quantum jump in communications world due to the solutions that supplied via this device, in addition to its capabilities that make it advanced to different conventional wi-fi systems.

\section{Increasing the Coverage}

Coverage vicinity is refers to the location where the communique among a user and the bottom station is to be had, because smart antennas is more directive than convention systems together with omnidirectional antennas or sectoring systems ,it could acquire higher insurance. This feature notably associated with higher benefit this is provided with the aid of the adaptive device. Moreover, it's been proved that this smart system achieves coverage more via (M) than traditional systems, while the variety of required base station has decreased by means of $(1 / \mathrm{M})$ through the use of clever antenna system with $\mathrm{M}$ antenna elements.

\section{Increasing Capacity}

Adaptive array gadget is able to meet the growing need for capacity via being able to radiates and receives in favored direction even as suppress the interference or noise that have an effect on the provider and restrict the possibility of re-use of frequency. Interference rejection characteristic enables the system to growth the sign-to-interference ratio which in turns boom the ability.

\section{Increasing bit-rate}

Depending on the spatial variation of the signals, the system can make the most this selection to reject signals coming from more than one paths, which purpose multipath fading and ISI [2]. Instead of using the equalizer to recover the Sign, adaptive array machine is used which will lessen the delay spread of the channel and that could reject multipath and assist the bit rate.

\section{Security}

The security function is taken into consideration a important difficulty within the area of communications to keep away from intruding on customers community records, the solution provided by the clever antenna lies in its 
capability to radiate in adaptive way with high gain; accordingly, the transmission of indicators isn't in all directions and thereby lowering the opportunity of spying on information and boom security while the hacker ought to be in the identical place of the customers [3].

\section{Antenna Array}

Transmit and receive range are powerful methods for exploiting the widespread blessings which might be to be had in more than one antenna structures like a couple of-input more than one-output (MIMO) wi-fi systems [4]. These advantages encompass however now not limited to range advantage (independent fading paths, channel variability discount), array gain (average signal-to-noise ratio growth, beam forming, the advantage is proportional to the array dimensions), multiplexing advantage (ability or records price linear increase), and interference gain (aggressive frequency reuse method, area-time sign processing to lessen the interference results).[10-12]

It's far widely known that the radiation pattern generated through a single-antenna detail is notably huge with low values of directivity and advantage and with much less manipulate skills over the critical parameters. Enlarging the antenna dimensions through assembling several radiating antenna factors (array) in geometrical and electric configurations ends in improved directive characteristics. The assembled antenna factors in any array may be same (same form of antennas like dipole, micro-strip, reflector, aperture, wave-guide, horn, and so forth.) or distinct. The entire radiation sample of the antenna array may be managed and formed using many methods which include [5-9]:

- The geometrical configurations (linear, planner, round, and so on.).

- The relative distance between the factors (vicinity and displacement).

- The amplitude and phase of the feeding electric modern for each antenna detail.

- The relative radiation pattern of the man or woman antenna detail.

The entire radiation pattern of the antenna array with same elements is received by using sample multiplication where the radiation sample of a unmarried detail located at a reference factor is accelerated by using the array element (AF).[12-15]

The ultimate point can be well supported and defined via an instance. Let us consider a linear antenna array with general wide variety of same elements equal to $M$ with uniform spacing (d) placed symmetrically along the equal axis as shown in Figure-3 (spherical coordinates with radial distance r, azimuth attitude $\varphi$, and elevation or polar angle $\theta$ ).

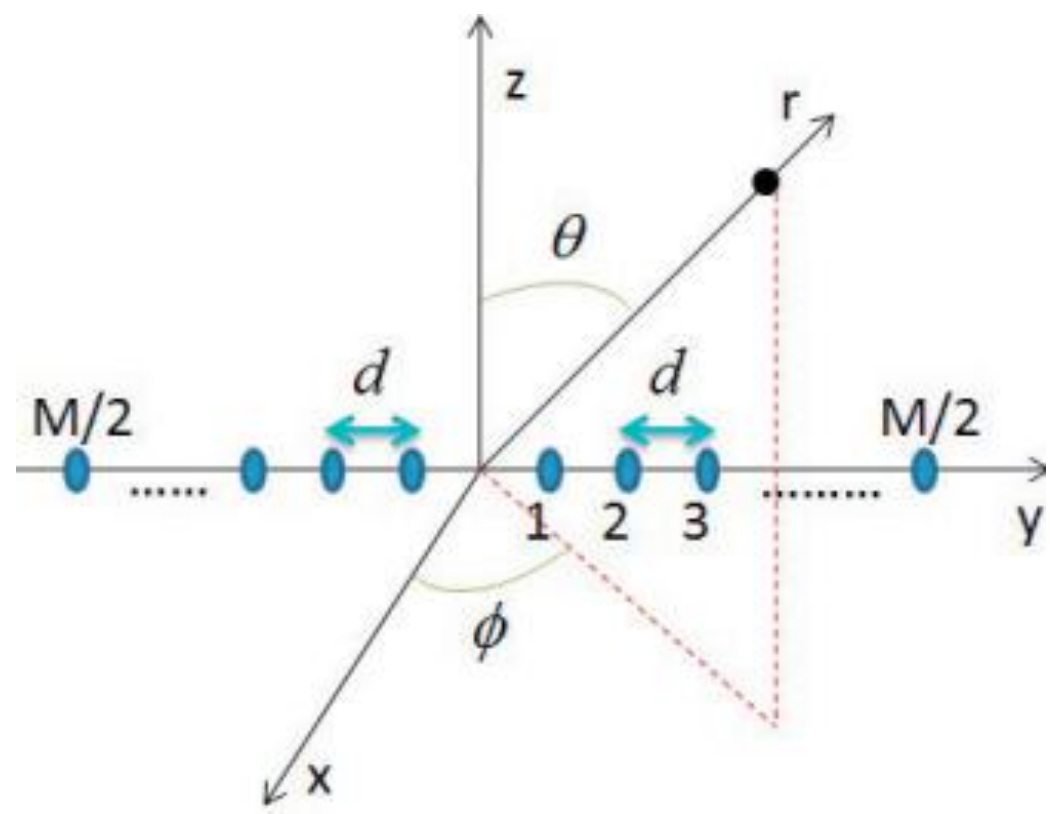

Fig-3: Linear Antenna Array with M no. of elements

\section{Adaptive Beam Forming (ABF)}

It separates both desired and interfering signal by adjusting weights by increasing Signal to noise ratio [SNR] and array output. Adaptive beam forming algorithms are formulated on the basis of some performances. These performing criteria includes maximum gain and variance minimum mean square error [MMSE], maximum signal to noise interference. Figure-4 shows type of adaptive beam forming algorithm. 


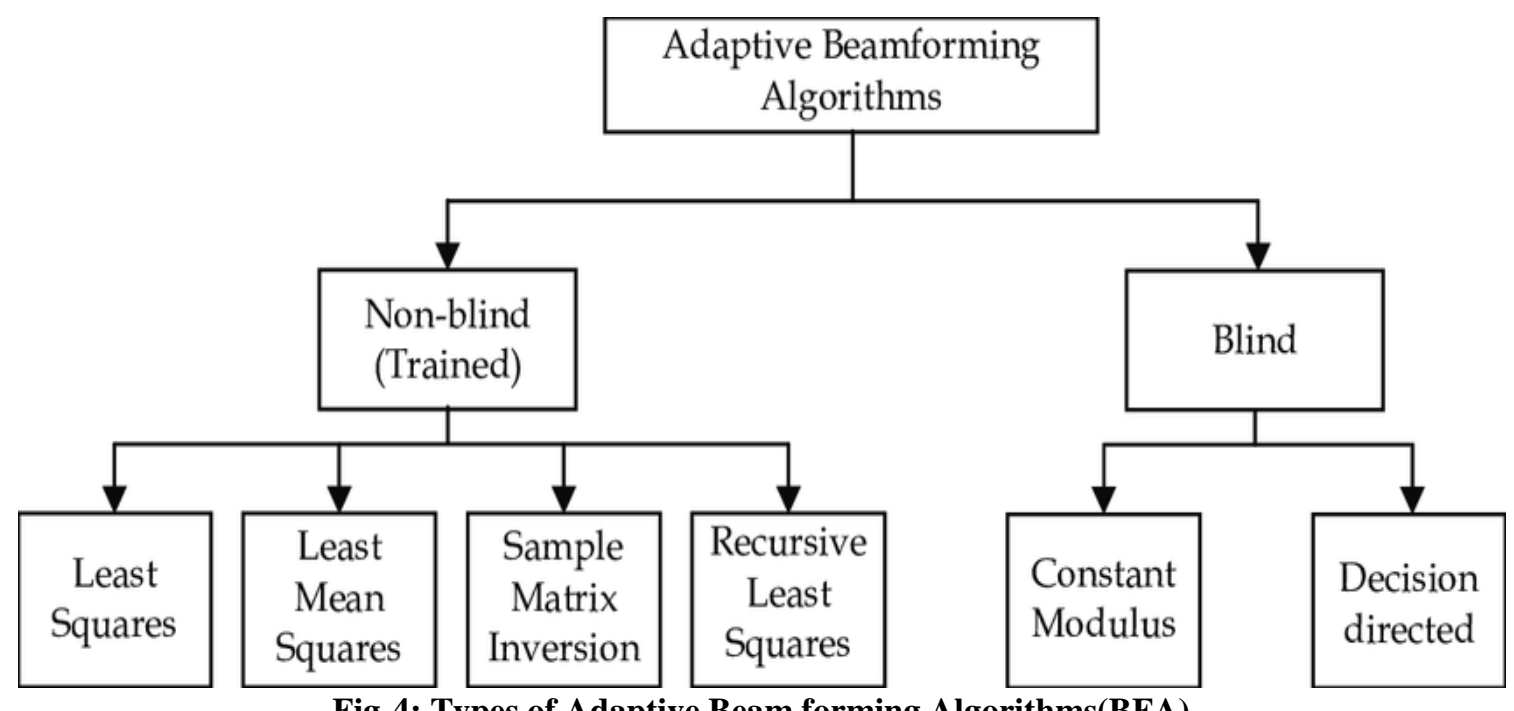

Fig-4: Types of Adaptive Beam forming Algorithms(BFA)

\section{Blind adaptive beam forming}

An adaptive algorithm which does not require an information about training sequence is known as blind adaptive beam forming. The use of training sequence has some requirements on the receiver side that can be difficult to meet in practical cases. Firstly, most important, the timing of training sequence must be known. i.e., even if the receiver knows that the transmitter transmits a sequence of training symbols periodically, but how will the receiver know when to expect it? secondly, carrier frequency should be known. In low signal-to-interference-plus-noise ratio (SINR) and multipath environment, carrier and timing synchronization are very difficult to achieve. In blind adaptive algorithm there is no requirement of carrier and timing synchronization[6-10]. Blind adaptive algorithm totally ignores the necessity of a training sequence. This algorithm is advisable since the use of a training sequence reduces the number of bits available for transmitting information. The two main classes of blind adaptive algorithms are:

1. Constant modulus algorithm [CMA]

2. Decision directed algorithm [DDA]

\section{Constant Modulus Algorithm [CMA]}

The CMA is conceivably the popular blind algorithm and is also used in different practical applications considering it does not require usage of transmitted signal exception in one case where the signal waveform which is transmitted has constant envelope. As it does not make use of training signal, it conserves lot of energy. CMA can be applied to non-constant modulus signals if the kurtosis is less than two i.e., to signals like PSK that have non-rectangular pulse shape.

CMA algorithm is used to lessen overhead of systems and also to decrease total output energy. As a consequence, number of bits for transforming information is increased which in return leads magnified capacity. This algorithm seeks for a signal with constant magnitude i.e., modulus within the received data vector. The received data vector consists of interference, noise with required signal. This is the reason why it can only identify one signal which has greatest power. CMA seeks a beam former weight vector which minimizes a cost function.

The weights are calculated using given equations:

$$
\mathrm{w}(\mathrm{n}+1)=\mathrm{w}(\mathrm{n})+\mu \mathrm{e}^{*}(\mathrm{n}) \mathrm{x}(\mathrm{n})
$$

error signal,

$$
\mathrm{e}(\mathrm{n})=(\mathrm{y}(\mathrm{n}) /|\mathrm{y}(\mathrm{n})|)-\mathrm{y}(\mathrm{n})
$$

Motive is to reduce the error e(n) between the signal output $y(n)$ and the reference signal $y(n), d(n)$ is known as hard limit of $\mathrm{y}(\mathrm{n})$ and given as:

$$
d(n)=e(n)+y(n)
$$

The signal from each element is multiplied with weights and are all combined linearly and is given as,

$$
\begin{aligned}
& \mathrm{y}(\mathrm{n})=\mathrm{w}^{\mathrm{H}}(\mathrm{n}) \mathrm{x}(\mathrm{n}) \ldots \ldots \ldots \ldots \ldots \ldots \ldots \text { (iv) } \\
& \mathrm{w}=\left[\mathrm{w}_{1}, \mathrm{w}_{2}, \mathrm{w}_{3}, \mathrm{w}_{4}, \ldots . \mathrm{w}_{\mathrm{n}}\right] ; \text { known as complex weight vector, }
\end{aligned}
$$


$\mathrm{x}(\mathrm{n})$ is an input signal

$\mathrm{H}$ is known as complex conjugate transpose or Hermitian response, $\mu$ is the step size or rate of adaptation used to control the convergence rate. CMA has broadest beam width in the desired direction. CMA impulsively chooses one or several of multipaths as desired signal. When array vector is updated it does not need to synchronize with received signal. It has limited applications as it is valid only for constant signals. One of the main drawbacks of the CMA is that, in an environment containing multiple constant modulus signals, a CMA beamformer will typically draw out the strongest signal which may not be the required signal. This can be overcome by collecting all the signals in the domain and then determining which is the required signal or another way is known as Multi-stage CMA.

\section{Decision Directed Algorithm [DDA]}

Decision-directed is one of the most natural approach in blind adaptive beam forming which can be bid to any digitally modulated signal. Here, data which is received from the transmitter is passed through linear combiner and then the output is demodulated. Decision directed algorithms uses methods of steepest-descent constant modulus algorithm. Based on the output of a threshold decision device a reference signal is generated. The CMA references signal $y(n) /|y(n)|$ can be replaced by a DD term given as $\operatorname{sgn}(\operatorname{Re}(\mathrm{y}(\mathrm{n})))$. This equation will be used by decision directed form on demodulated signal to testimonial the change in the modulus of output. The equation for DD is summarized as:

$$
\begin{aligned}
& w(n+1)=w(n)-1 / 2 \mu x(n) e^{*}(n) \\
& e(n)=y(n)-\operatorname{sgn}(\operatorname{Re}(y(n))) \ldots \ldots \ldots \\
& y(n)=w^{H}(n) x(n) \ldots \ldots \ldots \ldots \ldots \ldots
\end{aligned}
$$

Where, * is complex conjugate and sgn is signum function.

Many versions of DDA can be derived even including RLS, Normalized LMS, LMS. Unlike non-blind algorithm, the error in blind adaptive algorithm is calculated from the actual received signal and not from training sequence. Every so often a DDA is called as "finite alphabet" property of digitally modulated signals. This is because one finite set of possible value is occupied by a digital message i.e., signaling is drained from a finite alphabet.

\section{Non-Blind adaptive beam forming}

The second type of adaptive beam forming is non-blind adaptive beam forming as it requires information about training signal sequence i.e., it requires statistical understanding of the transmitted sequence signal in order to enhance array weights. The required training signal sequence is sent by the transmitter to the receiver during the training period. Non-blind adaptive beam forming consists of four classes:

1. Least mean square [LMS] algorithm.

2. Normalized LMS [NLMS] algorithm.

3. Sample matrix inversion [SMI] algorithm.

4. Recursive least square [RLS] algorithm.

\section{Least Mean Square [LMS]}

The Least Mean Square (LMS) algorithm is one of the most deep-rooted adaptive algorithms. LMS algorithm is also known as Stochastic Gradient Descent (SGD) algorithm. It is a simple and beam forming technique. Channel in a wireless communication vary with time, for this reason the weight vector must be changed periodically, so usage of minimum mean square error [MMSE] or least square [LS] technique is not commendable. Thus, LMS uses preceding solution to calculate succeeding weight set. LMS is a gradient based beam forming algorithm that contains repetitive process to make consecutive changes in the negative gradient direction which lastly results in minimum mean square error [MMSE]. The enhancement for LMS is done by employing the Steepest Descent Method which is a repetitive way of enhancing the array weights. The succeeding weight vector which minimizes cost function is given as:

$$
\mathrm{w}(\mathrm{n}+1)=\mathrm{w}(\mathrm{n})+\mu \mathrm{x}(\mathrm{n}) \mathrm{e}^{*}(\mathrm{n}) \ldots \ldots(\mathrm{viii})
$$

Where, $0 \leq \mu \leq 1 / 2 \lambda_{\max }$

$\mu$ is the step size which is always positive and controls the speed of adaptation. $\lambda_{\max }$ is the largest eigen value and is trace of auto-correlation matrix. LMS algorithm depends on three element step size parameter, eigen value of correlation matrix of the input data and number of weights.

As LMS algorithm uses principal of weight adaptation, LMS algorithm has least computational complexity, high stability with simple implementation but its applications are limited due to its slow convergence, sometimes it may require more than thousands of iterations for convergence. 


\section{Normalized LMS [NLMS]}

NLMS is modified version of LMS method, but offers much faster convergence with only a slightly higher computational load, this is because LMS is very sensitive to the scaling of its input vector $\mathrm{x}(\mathrm{k})$. LMS and NLMS offers same practical implementation. The NLMS update is given by:

$$
\begin{gathered}
\mathrm{w}(\mathrm{n}+1)=\mathrm{w}(\mathrm{n})+\left(\mu \mathrm{e}(\mathrm{n}) \mathrm{x}^{*}(\mathrm{n}) / \mathrm{x}^{\wedge} \mathrm{H}(\mathrm{n}) \mathrm{x}(\mathrm{n})\right) \\
\mathrm{OR} \\
\mathrm{w}(\mathrm{n}+1)=\mathrm{w}(\mathrm{n})+(1 /\|\mathrm{x}(\mathrm{n})\|)^{\wedge} 2 \mu \mathrm{x}(\mathrm{n}) \mathrm{e}^{*}(\mathrm{n}) \ldots \ldots
\end{gathered}
$$

Conjugate of input $x(n)$ is done by Hermitian transpose [H]. In NLMS algorithm, step size is said to be normalized because size parameter is dependent on input $\mathrm{x}(\mathrm{n})$ which results in greater stability with unknown signals. Normalized- LMS has better radiation pattern and more directed when implemented. NLMS maintains better composure between performance and simplicity. NLMS is better than LMS for correlated and whitened data but not productive in computational complexity i.e., offers higher computational load.

\section{Sample Matrix Inversion [SMI]}

In SMI algorithm, to obtain most favorable array weight vector we use Minimum Mean Squared Error (MMSE). Since, there is an absence of cross-correlation vector and true auto-correlation matrix, an estimation is done to overcome. This estimation of weights for nth block is given by:

$$
\mathrm{w}_{\text {SMI }}(\mathrm{n})=\left[\mathrm{X}_{\mathrm{N}}(\mathrm{n}) \mathrm{X}_{\mathrm{N}}{ }^{\mathrm{H}}(\mathrm{n})\right]^{-1} \mathrm{~d}^{*}(\mathrm{n}) \mathrm{X}_{\mathrm{N}}(\mathrm{n})
$$

SMI gives better performance in a discontinuous traffic but requires interfere number and their position to be constant during the time of block acquisition. Convergence of SMI algorithm is much faster than LMS as it employs direct matrix inversion. It improves convergence speed at the rate of singularity problem of correlation matrix and computational complexity. This algorithm's performance can be verified in multiple paths and multiple signal environment.

\section{Recursive Least Square [RLS]}

RLS algorithm uses LS method as a criterion for optimization. To find the filter coefficients that link to recursively producing least squares of error signal RLS algorithm is used. Unlike LMS, it is not steepest descent technique, but an iterative and approximate solution to a least square problem. This algorithm is opposite of the other algorithms which aim to reduce mean square error. RLS filters depend on themselves unlike MSE filters which are dependent on their statistics. The gradient step size in LMS is replaced with gain matrix R-1(n) at the nth iteration and the equation is given as:

$$
w(n)=w(n-1)-R-1(n) x(n) e^{*}(w(n-1))
$$

Where, $\mathrm{R}-1(0)=\delta-1 \mathrm{I}, \delta$ small positive constant and $\mathrm{I}$ the $\mathrm{N} \times \mathrm{N}$ identity matrix.

RLS has maximum signal strength in desired direction and therefore has the best formation of main lobe. It has narrowest beam width, total rejection of interference, more power in side lobes but at the cost of computational complexity. RLS is used when fast tracking of is signal is needed. As it is an approximate LS method, it bids good convergence rate than SGD methods. This algorithm has better null depth than SMI algorithm. No need for the inversion of matrices which results in saving computational power. It is faster than LMS and NLMS but comparatively more complex. This algorithm also has minimum BER and error signal magnitude for which it is used for implementation on Base Station Smart Antenna System.

\section{CONCLUSION}

From the above literature survey it's far concluded that, there are various adaptive beam forming algorithms given for smart Antennas which have capability to provide most gain in desired course neglecting the interference circumstance. Those algorithms are carried out and evaluated considering exceptional parameters such as radiation sample, mean square error, convergence fee below different conditions of wide variety of person, number of variety of antenna elements and element spacing. Most antenna advantage implies the higher gadget overall performance below the interference situations. Via using a couple of paths and multiple users it gives any other manner to make the device greater compatible. The MATLAB software used is greater user pleasant. Same system can be simulated using Simulink software program. In preference to the use of only smart antenna we will use smart antenna with cognitive radio and examine respective algorithms. 


\section{REFERENCE}

1. Chen, Y., Le-Ngoc, T., Champagne, B., \& Xu, C. (2004). Recursive least squares constant modulus algorithm for blind adaptive array. IEEE Transactions on Signal Processing, 52(5), 1452-1456.

2. Stevanovic, I., Skrivervik, A., \& Mosig, J. R. (2003). Smart Antenna Systems for Mobile Communications: FINAL REPORT. Laboratoired Electromagnetisme et d'Acoustique Ecole Polytechnique Federale de Lausanne.

3. Balanis, C. A., \& Ioannides, P. I. (2007). Introduction to smart antennas. Synthesis Lectures on Antennas, 2(1), 1175 .

4. Compton, R. T. Jr. (1988). Adaptive Antennas - Concepts and Performance. Prentice Hall. Englewood Cliffs, New Jersey.

5. Agee, B. (1986, April). The least-squares CMA: A new technique for rapid correction of constant modulus signals. In ICASSP'86. IEEE International Conference on Acoustics, Speech, and Signal Processing (Vol. 11, pp. 953-956). IEEE.

6. Devasis Pradhan, "Circular Patch With Circular Slit Patch Antenna Used For Ultra Wide Band Application.," International Journal Of Electrical, Electronics And Data Communication (vol. 5, no. 2, pp. 84-87)

7. Kim, T, Tens of Gbps Support with mm Wave Beamforming System for Next Generation Communications, IEEE GLOBECOM, pp. 3790-3795, 2013.

8. Roh, W, Performances and Feasibility of mm Wave Beamforming System in Cellular Environments, IEEE ICC, pp. 2010-2016, 2013.

9. Schmidt, R. O. Multiple emitter location and signal parameter estimation, IEEE Trans. Antennas and Propagation, 34(3), pp.276-280, 1986.

10. Roy, R, Kailath, T. ESPRIT - A subspace rotation approach to estimation of parameters of cisoid in noise. IEEE Trans. Acoustics, Speech and Signal Processing, 34(5), pp.1340-1342, 1986.

11. Liu, W. Blind adaptive wideband beamforming for circular arrays based on phase mode transformation, Digital Signal Processing, 21(2), pp. 239-247, 2011.

12. Yang N, Wang L, Geraci G, Elkashlan M, Yuan J, Di Renzo M. "Safeguarding 5G wireless communication networks using physical layer security". IEEE Communications Magazine. April 2015, Vol. 53(4): pp. 20-27.

13. Sun S, Rappaport TS, Heath RW, Jr., Nix A, Rangan S. "MIMO for millimeter-wave wireless communications: Beamforming, spatial multiplexing, or both?”. IEEE Communications Magazine. December 2014, Vol. 52(12): pp. 110-121.

14. Devasis Pradhan, "Massive MIMO Technique used for 5th Generation System with Smart Antenna," International Journal of Electrical, Electronics And Data Communication(IJEEDC) ( vol. 6, no. 7, pp. 81-87)

15. Devasis Pradhan, "Design of Extended Circular Patch with Rectangular Stub and Circular Slit Used For Ultra Wide Band Application (X-Band)," IOSR Journal of Applied Physics (IOSR-JAP) (vol. 11, no. 4, pp. 14-24.)

CITATION: Prathviraj Khande et al (2021). An overview of Algorithms for Adaptive Beam Forming (ABF) for Smart Antenna System (SAS). South Asian Res J Eng Tech, 3(1): 23-30. 